\section{Unknown syndrome: abnormal facies, hypothyroidism, and severe retardation: a second patient}

SUMmaRY In the November 1987 issue of this journal, Young and Simpson' presented a female infant with abnormal facies (microcephaly, blepharophimosis, small, low set, posteriorly rotated ears, bulbous nose, carp shaped mouth, and micrognathia), congenital heart abnormalities (large atrial and ventricular septal defects), congenital hypothyroidism, and severe global retardation.

We have observed a male newborn with a similar pattern of malformations (figs 1 and 2).

\section{History}

Prenatal. No cigarettes or alcohol. No medication. Hydramnios at five months.

Birth. Caesarean section (hydramnios and postmaturity of 42 weeks). Required resuscitation. Apgar scores 1 at one

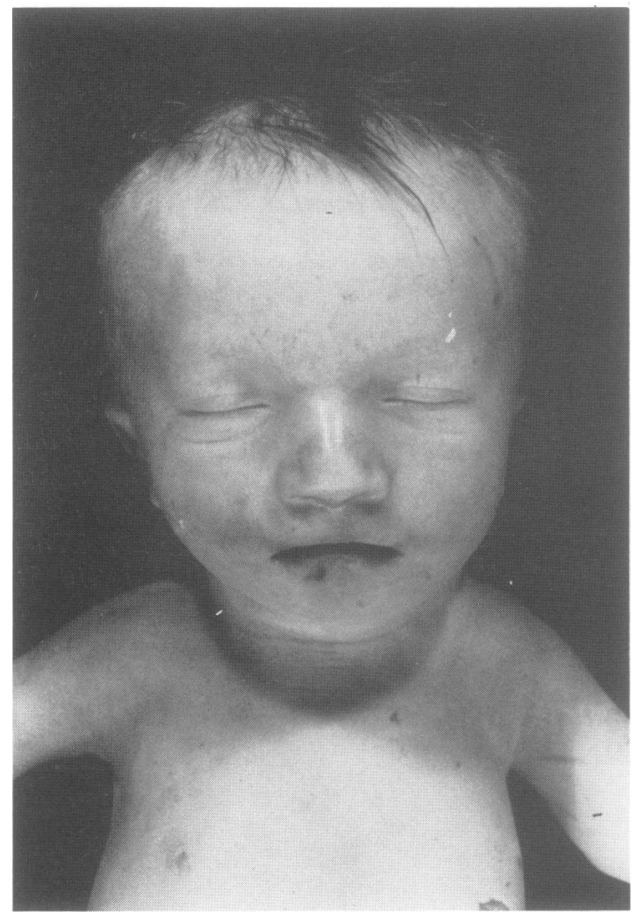

FIG 1 AP view of the dead neonate.

Received for publication 18 November 1987 Accepted for publication 22 December 1987. minute, 4 at 10 minutes. Despite intensive therapy death occurred after 12 hours.

Family. First child of healthy unrelated parents. Spon-들 taneous first trimester abortion in first pregnancy. Father $\overline{\frac{\omega}{5}}$ aged 34 years, mother 32 years.

\section{Clinical examination \\ Clinical examination}

Clinical postmortem findings. Weight $2600 \mathrm{~g}$ (3rd centile), $\overrightarrow{\vec{\omega}}$ length $46.5 \mathrm{~cm}$ (3rd centile), head circumference $35 \mathrm{~cm}$ (25th centile). Sloping forehead, prominent occiput, large posterior fontanelle, high nasal bridge with bulbous nasato tip, small palpebral fissures in normal position, thin upperĩ lip, low set ears with prominent antihelix, marked micro- or retrognathia, small tongue, cleft soft palate. bilateral ${ }_{+}^{-}$ simian crease, camptodactyly of fourth and fifth fingers, absence of distal interphalangeal creases, rockerbottom ${ }_{0}$ feet, lumbar hyperlordosis, bilateral cryptorchidism.

Internal findings. No internal malformations except for mobile caecocolon and absence of thyroid. No ectopic thyroid tissue. No gross malformations of the brain; en- $\vec{e}$ largement of the aqueduct of Sylvius and fourth ventricle. $\infty$

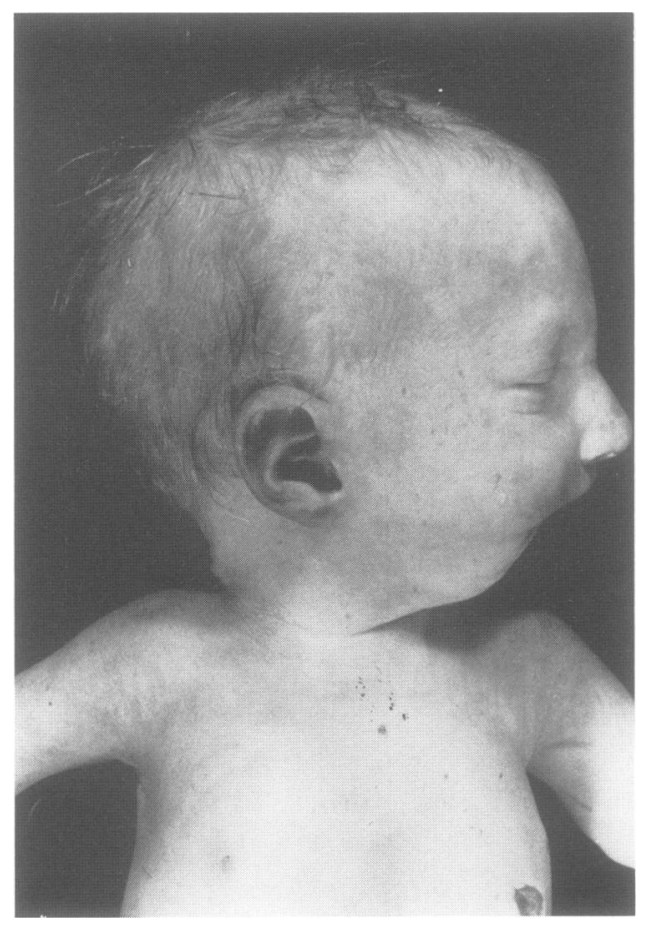

FIG 2 Lateral face. Note the sloping forehead, high nasal bridge, and marked microretrognathia. 
Karyotype. Normal G banded male karyotype on cord blood and skin biopsy. Normal parental karyotypes.

\section{Discussion}

This male neonate presents a number of striking similarities with the unknown syndrome presented by Young and Simpson. 'Among these similarities the most important seem to be the facial appearance with bulbous nose and microretrognathia and the hypothyroidism. The only major discordant finding is the absence of a cardiac anomaly in the present patient. Complete necropsy did not reveal other important internal malformations.

J P Fryns* and P Moermant "Centre for Human Genetics and †Department of Pathology, University of Leuven, Leuven, Belgium

\section{Reference}

1 Young ID. Simpson K. Unknown svndrome: abnormal facies. congenital heart defects. hypothyroidism, and severe retardation. J Med Genet 1987:24:715-6.

Correspondence and requests for reprints to Dr J P Fryns, Centre for Human Genetics, Herestraat 49, 3000 Leuven, Belgium.

\section{A further patient with the lethal type of Larsen syndrome}

SUMmaRY We present a female infant with multiple joint dislocations, flat facies, cleft soft palate, redundant neck skin, pulmonary hypoplasia, and skeletal abnormalities.

\section{History}

Prenatal. No medication, alcohol, or cigarettes. Normal liquor volume. Poor fetal movements.

Birth. Normal vaginal delivery at 42 weeks. Apnoeic after birth. No response to resuscitative measures and died at 30 minutes.

Family. Both parents healthy; 40 year old mother and unrelated 41 year old father. Two previous pregnancies had ended in spontaneous abortion at eight weeks and fetal death at 22 weeks. Necropsy in the latter case did not reveal any abnormality. The parents had a total of seven healthy children by previous partners.

\section{Clinical examination}

External (fig 1). Weight $2550 \mathrm{~g}$ (3rd to 10 th centile), length $45 \mathrm{~cm}$ (3rd centile), OFC $32 \mathrm{~cm}$ (3rd centile). Flat occiput, large posterior fontanelle and very narrow, diamond shaped anterior fontanelle. Low set ears, flat nasal bridge, and cleft soft palate. Short, broad neck owing to an excess of subcutaneous tissue. Cervical kyphosis. Dislocation of both hips and knees and talipes equinovarus deformity of the feet. Rhizomelic shortening of upper limbs. Bilateral single palmar creases.

Necropsy. Severely hypoplastic lungs, weight $13 \mathrm{~g}$ and $11 \mathrm{~g}$ (normal $25 \mathrm{~g}$ ), small spherical brain weighing $309 \mathrm{~g}$ with formation of microgyri in the frontal lobes. Fusion of frontal and parietal bones.

Radiology (fig 2). Hypoplasia of the vertebral bodies from $\mathrm{T} 2$ to T8. Severe hypoplasia of the fibulae, especially proximally.

\section{Discussion}

This patient has similar features to the two cases reported previously by Chen et al. ${ }^{1}$ The Larsen syndrome ${ }^{2}$ of multiple congenital dislocations associated with characteristic facial abnormality appears to arise as a result of a generalised mesenchymal disorder ${ }^{3}$ and collagen fibre abnormalities have been reported. This is a heterogeneous

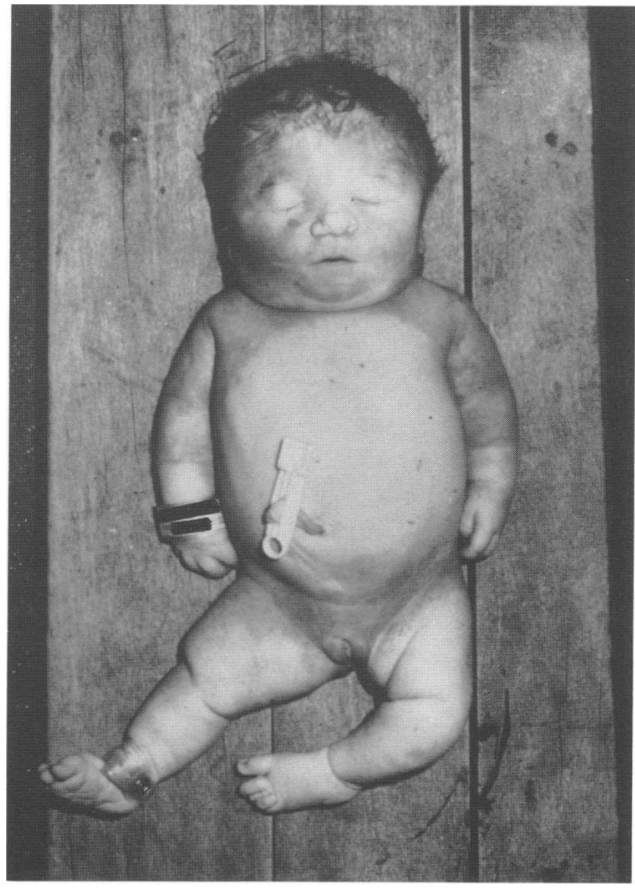

FIG 1 Postmortem view of the infant showing joint dislocations, unusual facies, and redundant neck skin. 